\title{
COMMENTARY
}

\section{Screening international travelers for hepatitis A antibodies versus routine vaccination: how generalizable are the results?}

Lee and Blodget's results indicate that selective prevaccination screening of travelers can lead to cost savings compared with routinely using hepatitis A vaccine. They concluded that screening travelers aged 51 years or older and those born in nonindustrialized countries would save about $\$ 25$ per traveler compared with routinely vaccinating all travelers. Aside from cost savings, travelers could benefit from screening for hepatitis A antibodies by needing fewer clinic visits and by avoiding adverse reactions to the vaccine.

Historically, many travel medicine clinics have been located within health departments and academic medical centers. Over time, primary care physicians and managed care organizations have also become sources of travel medicine services. As the authors imply, their study conducted within an HMO should be examined more closely to assess the representativeness of its patients and the ability to extrapolate the results to other travel medicine groups. Several questions immediately come to mind. First, what if hepatitis A vaccine costs are substantially lower than assumed by Lee and Blodget? Health departments and other state or federally administered travel medicine clinics generally are able to purchase vaccines at the lowest possible cost. Second, what if patient demographics vary markedly from those reported? For example, travel medicine patients attending a university-based clinic

Table Cost comparison of prevaccination screening strategies for hepatitis $A$ antibodies among international travelers*

\begin{tabular}{|c|c|c|c|}
\hline Screening strategy & Study results & $\begin{array}{l}\text { Cost } \\
\text { Scenario 1t }\end{array}$ & Scenario $2 t$ \\
\hline No screening; vaccinate all patients & 124.73 & 38.83 & 38.83 \\
\hline Screen all patients & 102.99 & 40.91 & 41.44 \\
\hline Screen patients $18-50$ years of age & 117.11 & 40.02 & 41.11 \\
\hline Screen patients born after 1945 in an industrialized country & 126.21 & 41.94 & 46.45 \\
\hline Screen patients born after 1945 in a nonindustrialized country & $115 \cdot 58$ & 36.86 & 38.06 \\
\hline Screen patients 51 years of age or older & 110.63 & 39.72 & 39.17 \\
\hline Screen patients born before 1945 in an industrialized country & 116.87 & 40.91 & 39.71 \\
\hline Screen patients born before 1945 in a nonindustrialized country & 121.03 & 38.07 & 38.63 \\
\hline $\begin{array}{l}\text { Screen patients } 51 \text { years of age or older or born after } 1945 \text { in a } \\
\text { nonindustrialized country }\end{array}$ & 99.58 & 37.16 & 38.40 \\
\hline Screen patients who previously traveled to an area of endemic hepatitis A & 103.01 & 39.11 & 39.11 \\
\hline Screen patients born in a nonindustrialized country & 111.89 & 36.09 & 37.87 \\
\hline
\end{tabular}

*Results represent total cost per traveler in dollars.

See accompanying text for assumptions and methods.

¥This strategy was not presented in the study but results were computed using the reported data.

John C Licciardone

Department of Family Medicine

University of North Texas Health Science Center

3500 Camp Bowie Blvd Fort Worth, TX 76107

Correspondence to: Dr Licciardone

jlicciar@hsc.unt.edu

Competing interests: None declared

West I Med 2000;173:329-330 
in the Southwest were younger than the study patients. ${ }^{1}$ Third, what if fewer travelers are born in nonindustrialized countries than observed in the study?

Sensitivity analysis represents 1 approach to addressing these issues. ${ }^{2}$ Although a comprehensive sensitivity analysis is not my intent, the table presents a comparison of the study results and 2 alternative scenarios. The first scenario represents a 1-way sensitivity analysis using the assumption of low vaccine cost to determine if hepatitis A antibody screening strategies remain preferable to routinely vaccinating all travelers (the 2 -dose vaccine series may be purchased by a travel medicine clinic in the Southwest for slightly less than $\$ 33$ vs about $\$ 119$ in the study). Using higher vaccine costs than in the study would augment the cost savings attributed to the various screening strategies. The second scenario represents 1 aspect of a 3-way sensitivity analysis, again purposely making assumptions to test the robustness of the study results regarding cost savings attributed to screening for hepatitis A antibodies. The assumptions in this scenario are low vaccine cost (described above), a younger patient distribution ${ }^{1}(22.7 \%$ older than 50 years vs $59.8 \%$ in the study), and a lower prevalence of travelers born in nonindustrialized countries $(5.0 \%$ vs $14.2 \%$ in the study). In both scenarios, the remaining study variables and assumptions were unaltered and the percentages of positive hepatitis A antibody tests in each screened subgroup were assumed to remain unchanged. In addition, the strategy of screening only patients born in a nonindustrialized country was also tested.

Several findings are evident in the table. Both alternative scenarios resulted in substantially lower costs for all strategies, and the cost differences between strategies were greatly attenuated. Vaccinating all patients without screen- ing costs less than most screening strategies in both scenarios. Interestingly, the strategy associated with the greatest cost savings was screening only patients born in a nonindustrialized country. Screening patients born after 1945 in an industrialized country consistently costs more than any other strategy.

What does this mean for physicians who counsel travelers? It certainly appears that screening for hepatitis A antibodies in various subgroups of patients may reduce the costs and possible risks associated with routinely vaccinating patients. Nevertheless, the cost savings may be substantially smaller than reported in the study, particularly in health departments or in other government-affiliated clinics. The heterogeneity of travelers across clinics and the many variables to be considered in cost-comparison studies suggest that a universally acceptable hepatitis A screening strategy will remain elusive. Regardless of whether screening is contemplated, it is imperative that travelers be advised to seek counseling at least 1 month before departure to ensure adequate vaccination for hepatitis A or other travel-related diseases, if needed.

Dr Licciardone is medical director of the International Travel Medicine Clinic at the University of North Texas Health Science Center at Fort Worth.

\section{References}

1 Licciardone JC, Herron KM, Marshall MA, Woodworth RM. Factors associated with the vaccination of international travelers: a multivariate analysis. J Am Osteopath Assoc 2000;100:423-424, 427-431.

2 Petitti DB. Meta-Analysis, Decision Analysis, and Cost-Effectiveness Analysis: Methods for Quantitative Synthesis in Medicine. 2nd ed. New York: Oxford University Press; 2000.

\section{ANY QUESTIONS?}

Do you have a clinical question you'd like to see answered? If so, here's your chance to get a curbside consult from our expert team, which includes many of the top clinicians in the West.

\section{ANY ANSWERS?}

Maybe you have strong views about something you read in this issue-something we got wrong perhaps? Or do you have further clinical experience you'd like to share? Perhaps you have suggestions for new topics you'd like to see us address from an evidence-based perspective.

Whatever questions, comments, or other contributions you have, we'd like to receive them. We realize that it's experience like yours that makes the journal come alive. Please send your questions, ideas, or comments to us by email:wjm@ewjm.com. 